\title{
Preparation and Antidiabetic Effect of Orally Administered Nifedipine-Loaded Solid Lipid Nanoparticles in Fructose-Induced Diabetic Rats
}

\author{
Sabarni Sarker', Md. Ashraf Ali², Ranjan Kumar Barman³, Shuji Noguchi, Yasunori Iwao5, \\ Shigeru Itai ${ }^{5}$, Mir Imam Ibne Wahed ${ }^{3 *}$
}

\footnotetext{
${ }^{1}$ Department of Pharmacy, Faculty of Life and Earth Sciences, Jagannath University, Dhaka, Bangladesh

${ }^{2}$ Department of Pharmacy, Mawlana Bhasani University of Science of Technology, Tangail, Bangladesh

${ }^{3}$ Department of Pharmacy, Faculty of Science, University of Rajshahi, Rajshahi, Bangladesh

${ }^{4}$ Laboratory of Pharmaceutics, Faculty of Pharmaceutical Sciences, Toho University, Tokyo, Japan

${ }^{5}$ Department of Pharmaceutical Engineering and Drug Delivery Science, School of Pharmaceutical Sciences,

University of Shizuoka, Shizuoka, Japan

Email: *drmirimam@gmail.com
}

How to cite this paper: Sarker, S., Ali, M.A., Barman, R.K., Noguchi, S., Iwao, Y., Itai, S. and Wahed, M.I.I. (2018) Preparation and Antidiabetic Effect of Orally Administered Nifedipine-Loaded Solid Lipid Nanoparticles in Fructose-Induced Diabetic Rats. Pharmacology \& Pharmacy, 9, 457-471. https://doi.org/10.4236/pp.2018.910035

Received: September 17, 2018

Accepted: October 27, 2018

Published: October 30, 2018

Copyright (c) 2018 by authors and Scientific Research Publishing Inc. This work is licensed under the Creative Commons Attribution International License (CC BY 4.0).

http://creativecommons.org/licenses/by/4.0/

\begin{abstract}
The use of Nifedipine (NI), a dihydropyridine calcium channel blocker, is limited due to its poor aqueous solubility. However, NI loaded solid-lipid nanoparticles (NI-SLN) are known to exhibit suitable pharmacokinetic properties and good biocompatibility. The present investigation was designed to evaluate the effects of NI-SLN on glucose homeostasis, lipid metabolism and liver function in fructose-induced diabetic rats. NI-SLN was prepared by high pressure homogenization technique followed by lyophilization with trehalose as cryoprotectant. Diabetes was induced into rats by the administration of fructose $(10 \%)$ in drinking water for six weeks. After induction of diabetes, rats were divided into four groups for the oral ingestion of NI, NI-SLN and/or vehicles and their effects on blood glucose levels, oral glucose tolerance test (OGTT), lipid profile, biochemical parameters, electrolytes and histopathology were observed. Single dose administration and treatment with NI-SLN showed significant glucose lowering efficacy in fructose-induced diabetic rats. Although NI and NI-SLN did not alter the fasting blood glucose level in normal rats, diabetic rats treated with NI-SLN resulted in significant reduction in glucose level for $24 \mathrm{hr}$. In OGTT, NI-SLN exhibited significant antihyperglycemic activity in both normal and diabetic rats. So, NI-SLN has better glucose lowering efficacy than that of pure NI in diabetic rats. The survival rates in rats among the treatment groups were $100 \%$. Treatment with
\end{abstract}


NI-SLN significantly improved lipid profiles than NI alone and the effect was dose-dependent. Administration of NI-SLN significantly reduced uric acid, creatinine levels and maintained a good cationic balance. After two weeks of NI-SLN treatment, hepatocytes regained their normal architecture, and the beneficial effect could be correlated with the reduction of SGOT and total bilirubin levels. Therefore, NI-SLN was found to be useful for the enhancement of bioavailability and exhibited profound antidiabetic activity in rats. The results of the study suggested that NI-SLN exerted better improvement in glucose levels, lipid profiles and organ protection than pure NI and might have some beneficial effects in the management of diabetic patients.

\section{Keywords}

Formulation, Solid Lipid Nanoparticle, Calcium Channel Blocker, Nifedipine, Fructose, Diabetes

\section{Introduction}

A large number of active pharmaceutical ingredients used in pharmaceutical formulations are practically insoluble in water [1]. This hydrophobic nature of drugs leads to poor systemic bioavailability, which in turn, may also be affected by insufficient permeation of drug through the gastrointestinal membrane. In particular, for drugs that undergo extensive first-pass metabolism, the scenario is often worsening. Presently, the development of different technologies to improve solubility remains the mainstay in pharmaceutical research.

Calcium channel blockers (CCBs) are a class of vasodilators widely used in the treatment of hypertension. The therapeutic acceptability of CCBs varies depending on their pharmacokinetic and pharmacodynamic properties [2]. Nifedipine (NI), a prototype dihydropyridine $\mathrm{CCB}$, belongs to Class II of Biopharmaceutics Classification System (BCS) of drugs and is characterized by its poor water solubility but high membrane permeability. Generally, NI is associated with a poor bioavailability, short half-life and significant fluctuations in plasma drug concentrations were also observed with this drug [3]. By modifying the formulations of NI, it is possible to maintain the plasma drug concentration at the desired level with minimal fluctuations which would provide a prolonged therapeutic effect with a reduced incidence of side effects [4]. Recently, our co-workers prepared nifedipine-loaded solid lipid (NI-SLN) lyophilized with trehalose by high pressure homogenization of NI and hydrogenated soybean phosphatidylcholine (HSPC) and dipalmitoylphosphatidylglycerol (DPPG); and developed a stable and lyophilized NI-SLN having 4-fold better water solubility than that of pure NI [5] [6] [7]. Further, the newly developed NI-SLN could play a significant role in the management of diabetes and hypertension.

Generally, hyperinsulinemia, insulin resistance, hypertriglyceridemia and obesity are treated by hypoglycemic (such as metformin) and lipid-lowering agents. 
Recent studies indicated that insulin sensitivity is improved by several different classes of antihypertensive agents such as angiotensin-converting enzyme inhibitors (ACE-Is), angiotensin-II receptor blockers (ARBs) and peripheral $\alpha$-adrenergic antagonists [8] [9]. CCBs are used as major therapeutic agents in the treatment of systemic hypertension and ischemic heart disease. However, significant variation in their effects on carbohydrate and lipid metabolism has been reported. In contrast to diuretics and $\beta$-blockers (that worsen insulin sensitivity), CCBs have generally been considered to be metabolically neutral (verapamil, diltiazem) or slightly positive (isradipine) in their effects on glucose and insulin [10] [11]. However, it was reported that NI treatment suppressed plasma glucose, insulin and triglyceride in patients with hypertension [12]. The objective of this study was to evaluate the effects of newly developed NI-SLN on plasma glucose, lipid profiles and liver functions in fructose-induced diabetic rats.

\section{Methods and Materials}

\subsection{Drugs and Chemicals}

Fructose was purchased from Merck, Germany. Serum concentration of total cholesterol (TC), triglycerides (TG), high-density lipoprotein (HDL), creatinine, uric acid, calcium and serum glutamate oxaloacetate transaminase (SGOT) were colorimetrically determined by commercial diagnostic kits (Human, Germany). Total bilirubin was determined using commercial kit obtained from Randox, UK. To produce lyophilized NI-SLN, HSPC (COATSOME NC-21) and DPPG (COATSOME ${ }^{\oplus}$ MGLS-6060) were collected from Nippon Oil and Fats Co., Ltd. (Japan). Trehalose was bought from Wako Pure Chemical Industries Ltd. (Japan). Pure NI was a generous donation by Square Pharmaceuticals Ltd., Pabna, Bangladesh. All other chemicals and solvents used were of analytical grade.

\subsection{Preparation of NI-SLN}

The preparation of NI-SLN was performed according the methods of Barman et al. as described earlier [5]. Briefly, for preparation of the NI-SLN suspension, 40 $\mathrm{mg}$ of NI and $1000 \mathrm{mg}$ of HSPC and DPPG in 5:1 molar ratio were physically mixed in a mortar for $5 \mathrm{~min}$. Then, the mixture was co-grounded by a roll mill (R3-1R, Kodaira Seisakusho Co., Ltd.). The grinding part consisted of three rollers of which the rotating velocity ratios were fixed as 1:2.5:5.8. Grinding was carried out for $5 \mathrm{~min}$. During grinding, most of the samples were adhered to the rollers, and some partially fell down from the rollers. Therefore, the mill was stopped every $30 \mathrm{sec}$ to collect the material. The co-grinding cycle was repeated 10 times. The resultant roll mixture was dispersed in $200 \mathrm{ml}$ of de-ionized distilled water and premixed using a Speed Stabilizer (10,000 rpm, Kinematica Co.) at $9000 \mathrm{rpm}$ for $10 \mathrm{~min}$, followed by high pressure homogenization (max pressure: $200 \mathrm{MPa}$, Nanomizer, NM2-L200-D10; Yoshida Kikaikogyo, Co.) with a pass cycle of 100. The suspension thus obtained was filtered through a $0.2 \mu \mathrm{m}$ membrane filter and stored in a refrigerator at $4^{\circ} \mathrm{C}$ for further use. To prepare 
lyophilized SLNs, $2 \mathrm{ml}$ of the NI-SLN suspension was collected into small vials containing $20 \mathrm{mg}$ of trehalose. Each vial was frozen at $-40^{\circ} \mathrm{C}$ for $3 \mathrm{hrs}$ and then the frozen sample was freeze-dried in a glass chamber for $24 \mathrm{hr}$ using a vacuum pump accompanied by a vapor condenser $\left(-20^{\circ} \mathrm{C}, 0.0225 \mathrm{Torr}\right)$. Then, secondary drying was carried out at $20^{\circ} \mathrm{C}$ for $24 \mathrm{hr}$.

\subsection{Animals}

Six-week old male Wister rats (100 - $120 \mathrm{~g}$ ) were purchased from Animal Center, Department of Pharmacy, Jahangirnagar University, Savar, Dhaka, Bangladesh. The rats were kept in animal cages under standard environmental conditions $\left(22^{\circ} \mathrm{C}-25^{\circ} \mathrm{C}\right.$, humidity $60 \%-70 \%, 12 \mathrm{hr}$ light: $12 \mathrm{hr}$ dark cycle). The rats were acclimated for 2 weeks and given standard rodent chow and water ad libitum. Throughout the study, the animals were cared in accordance with the guidelines for animal experimentation. The experimental protocol was approved by Institutional Animal, Medical Ethics, Biosafety and Biosecurity Committee (IAMEBBC) at the Institute of Biological Sciences, University of Rajshahi, Bangladesh.

\subsection{Experimental Design}

\subsubsection{Induction of Diabetes}

Diabetes was induced into normal rats after feeding fructose in drinking water (10\% solution) for 6 weeks [13] [14]. After overnight fasting, plasma glucose levels were measured by glucometer (One Touch Ultra, Japan) using blood sample from tail-vein of rat. Blood sugar levels higher than $5.6 \mathrm{mmol} / \mathrm{L}$ were considered as diabetic.

\subsubsection{Effect of NI-SLN on Blood Sugar Levels}

Both normal and diabetic rats were divided into three groups and subjected for the single dose oral administration of NI $(0.1 \mathrm{mg} / \mathrm{kg})$, NI-SLN $(0.1 \mathrm{mg} / \mathrm{kg})$ and/or vehicle (HSPC+DPPG, 5:1). The glucose levels of the rats were measured at $0 \mathrm{hr}$, before administration and after $0.5,1,1.5,2,3,4,5,6,7,8,16$ and 24 hours of administration of drugs and/or vehicle.

\subsubsection{Oral Glucose Tolerance Tests (OGTT)}

To perform OGTT, blood glucose levels of both normal and diabetic rats were measured after overnight fasting. After $30 \mathrm{~min}$ of oral administration of NI (0.1 $\mathrm{mg} / \mathrm{kg}$ ), NI-SLN $(0.1 \mathrm{mg} / \mathrm{kg}$ ) and/or vehicle (HSPC+DPPG, 5:1), a glucose load $(2 \mathrm{~g} / \mathrm{kg})$ was given to rats from both normal and diabetic group. Blood samples were withdrawn from the tail-vein of rats at $0 \mathrm{~min}$, before and 30, 60, 90 and 120 min after glucose loading. Further, blood glucose levels were estimated using glucose oxidase and peroxidase method [15].

\subsubsection{Treatment Protocol}

After induction of diabetes, rats were divided into five groups for the oral administration of NI, NI-SLN and/or vehicle (HSPC+DPPG, 5:1) into rats by gas- 
tric gavage once daily for 15 days. The groups were as follows: 1) Group DC (diabetic control, received vehicle); 2) Group NI (diabetic rats treated with pure NI $0.1 \mathrm{mg} / \mathrm{kg}$ ); 3) Group NI-SLN 0.01 (diabetic rats treated with NI-SLN 0.01 $\mathrm{mg} / \mathrm{kg}$ ); 4) Group NI-SLN 0.1 (diabetic rats treated with NI-SLN $0.1 \mathrm{mg} / \mathrm{kg}$ ); and 5) Group NI-SLN 1.0 (diabetic rats treated with NI-SLN $1.0 \mathrm{mg} / \mathrm{kg}$ ). Age-matched healthy rats were used as normal control (Group NC, received vehicle) and each group comprised of five animals.

The body weights and fasting blood glucose levels of rats from each group were estimated on 0 day, before and 5, 10 and 15 days after oral administration of drugs and/or vehicles. At the end of experiments, the rats were anesthetized, chest opened and blood samples were withdrawn directly from abdominal aorta at retroperitoneal space and poured into blood collecting tube [16]. The blood samples were centrifuged at $4000 \mathrm{rpm}$ for 10 minutes (Centurion, UK) and the serum samples were stored in refrigerator at $-40^{\circ} \mathrm{C}$ until further biochemical estimation. After sacrificing, liver, pancreas, heart and kidney were removed and cleaned of the surrounding tissues. The organ weights were measured immediately and the ratio of organ weights to body weights $(\mathrm{g} / \mathrm{kg})$ were calculated. Then, the parts of liver were stored in $10 \%$ formalin for histopathology.

\subsection{Biochemical Estimations}

The serum concentration of TC, TG and HDL were measured colorimetrically by photometric colorimeter (ERMA Inc., Japan) using commercially available wet reagent diagnostic kits (Human, Germany). Serum low density lipoprotein (LDL) was calculated by using Friedewald formula [17]:

$$
L D L=T C-\left(H D L+\frac{T G}{5}\right)
$$

Serum uric acid, creatinine, calcium and SGOT levels were determined by colorimetric method using a blood analyzer (Humalyzer, Germany). Quantitative measurement of serum electrolytes $\left(\mathrm{Na}^{+}, \mathrm{K}^{+}, \mathrm{Cl}^{-}\right)$was done by using an electrolyte analyzer (Erba Mannheim, Germany). Serum total bilirubin was determined by photometric method using commercial kit (Randox, UK).

\subsection{Histopathological Study}

The histopathological studies of liver were carried out at the Department of Pathology, Rajshahi Medical College, Rajshahi, Bangladesh. Briefly, livers from each group were fixed in phosphate buffered saline containing $10 \%$ formalin. The tissues were washed in running tap water, dehydrated in the descending grades of isopropanol and finally, cleared in xylene. The tissues were then embedded in molten paraffin wax. After embedding in paraffin, several transverse sections $(5 \mu \mathrm{m})$ were cut from the mid organ level by microtome machine and stained with hematoxylin-eosin (HE). The specimens were observed under light microscope $(400 \times$ magnification) with digital camera (Optika, Italy) for any structural changes. 


\subsection{Statistical Analysis}

Data were expressed as mean \pm standard error of mean (SEM). Student's t-test was used for comparison between two groups. Comparisons among more than two groups were performed by one way analysis of variance (ANOVA) followed by Tukey's post-test. The differences were considered as significant when $\mathrm{p}<$ 0.05. All statistical analysis was performed by MS Office Excel Analysis ToolPak and GraphPad Prism software.

\section{Results}

\subsection{Hypoglycemic Effect of NI-SLN in Normal and Diabetic Rats}

The effects of NI-SLN on blood glucose levels in normal and diabetic rats for 24 hr are shown in Table 1. The normal rats treated with NI and NI-SLN (single dose) did not alter the fasting blood glucose level after $0.5,1,1.5,2,4,6,8,16$ and $24 \mathrm{hr}$ of oral administration when compared to $0 \mathrm{hr}$ level. The blood glucose levels were higher in diabetic rats. However, diabetic rats treated with NI-SLN resulted in significant reduction in glucose level up to $24 \mathrm{hr}$ in which most prominent effects were observed between $1-2 \mathrm{hr}(\mathrm{p}<0.01)$ as compared to that of $0 \mathrm{hr}$ level. In contrast, NI treated diabetic rats showed a reduction in fasting glucose level between 4 - $6 \mathrm{hrs}$ but the effect was not significant. So, NI-SLN has shown better glycemic control than that of pure NI in diabetic rats.

\subsection{Effect of NI-SLN on OGTT}

The effects of NI-SLN on OGTT in normal and diabetic rats are shown in Figure 1. In NC rats, the blood glucose levels were peaked after $30 \mathrm{~min}$ and remained high up to $60 \mathrm{~min}$. Although NI reduced blood glucose levels, NI-SLN exhibited significant antihyperglycemic activity in normal rats between $30-60 \mathrm{~min}(\mathrm{p}<$ 0.05). In DC rats, blood glucose concentration were peaked after $30 \mathrm{~min}$ and remained high over the next 90 min. Rats in NI and NI-SLN group showed a significant attenuation in blood glucose levels after 30, 60, 90 and 120 min when compared with DC rats.

\subsection{Treatment Protocol}

\subsubsection{Clinical Course}

The survival rates in rats among the treatment groups were $100 \%$. None of the rats died in NI-SLN, NI and vehicle-treated groups. No sign of physiological and pathological changes were observed in rats treated with drugs and/or vehicles.

\subsubsection{Time Course of Changes in Blood Glucose Levels}

Time course of changes in blood sugar levels are shown in Table 2. On day 0 , before the initiation of treatment the blood glucose levels were significantly higher in diabetic rats compared to NC rats $(\mathrm{p}<0.01)$. Oral administration of NI-SLN significantly lowered the blood glucose levels on day 5, 10 and 15; and the effect was dose-dependent. Although NI lowered glucose levels in diabetic 
Table 1. Effects of NI-SLN on blood glucose levels in normal and fructose-induced diabetic rats.

\begin{tabular}{ccccccccccc}
\hline Group & \multicolumn{7}{c}{ Mean Blood Glucose Level (mmol/L) } \\
\hline Normal & $0 \mathrm{hr}$ & $0.5 \mathrm{hr}$ & $1 \mathrm{hr}$ & $1.5 \mathrm{hr}$ & $2 \mathrm{hr}$ & $4 \mathrm{hr}$ & $6 \mathrm{hr}$ & $8 \mathrm{hr}$ & $16 \mathrm{hr}$ & $24 \mathrm{hr}$ \\
\hline NC & $5.5 \pm 0.12$ & $5.6 \pm 0.23$ & $5.6 \pm 0.23$ & $5.7 \pm 0.39$ & $5.4 \pm 0.29$ & $5.1 \pm 0.49$ & $5.0 \pm 0.15$ & $5.1 \pm 0.23$ & $5.0 \pm 0.38$ & $4.8 \pm 0.17$ \\
NI & $5.8 \pm 0.15$ & $5.9 \pm 0.15$ & $5.6 \pm 0.03$ & $5.6 \pm 0.16$ & $5.7 \pm 0.38$ & $6.0 \pm 0.33$ & $6.0 \pm 0.17$ & $5.8 \pm 0.15$ & $5.9 \pm 0.12$ & $6.0 \pm 0.35$ \\
NI-SLN & $5.6 \pm 0.35$ & $5.8 \pm 0.12$ & $5.4 \pm 0.32$ & $5.2 \pm 0.06$ & $5.0 \pm 0.23$ & $5.5 \pm 0.29$ & $5.7 \pm 0.35$ & $5.6 \pm 0.49$ & $5.8 \pm 0.15$ & $5.5 \pm 0.26$ \\
\hline & & & & & Fructose & & & & & \\
\hline DC & $9.0 \pm 0.32$ & $8.9 \pm 0.49$ & $9.2 \pm 0.12$ & $9.4 \pm 0.35$ & $9.3 \pm 0.37$ & $9.0 \pm 0.23$ & $8.8 \pm 0.12$ & $8.9 \pm 0.32$ & $9.4 \pm 0.23$ & $9.8 \pm 0.46$ \\
NI & $8.5 \pm 0.17$ & $8.4 \pm 0.55$ & $8.1 \pm 0.26$ & $7.7 \pm 0.43$ & $7.8 \pm 0.23$ & $8.2 \pm 0.12$ & $8.3 \pm 0.29$ & $8.8 \pm 0.23$ & $9.3 \pm 0.26$ & $9.4 \pm 0.32$ \\
NI-SLN & $8.8 \pm 0.23$ & $8.0 \pm 0.58$ & $6.7 \pm 0.15^{\mathrm{b}}$ & $5.8 \pm 0.26^{\mathrm{b}}$ & $6.3 \pm 0.34^{\mathrm{b}}$ & $7.5 \pm 0.29^{\mathrm{a}}$ & $7.3 \pm 0.3^{\mathrm{a}}$ & $7.4 \pm 0.40^{\mathrm{a}}$ & $7.3 \pm 0.26^{\mathrm{a}}$ & $7.1 \pm 0.30^{\mathrm{a}}$
\end{tabular}

The results are expressed as means \pm SEM. ${ }^{a} \mathrm{p}<0.05$, and ${ }^{\mathrm{b}} \mathrm{p}<0.01$ versus $0 \mathrm{hr}$.

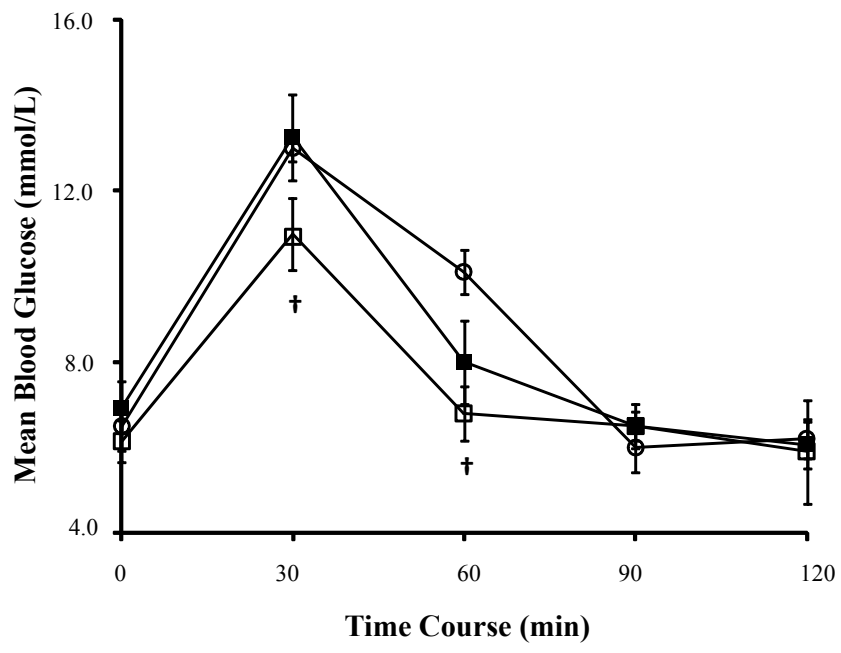

(a)

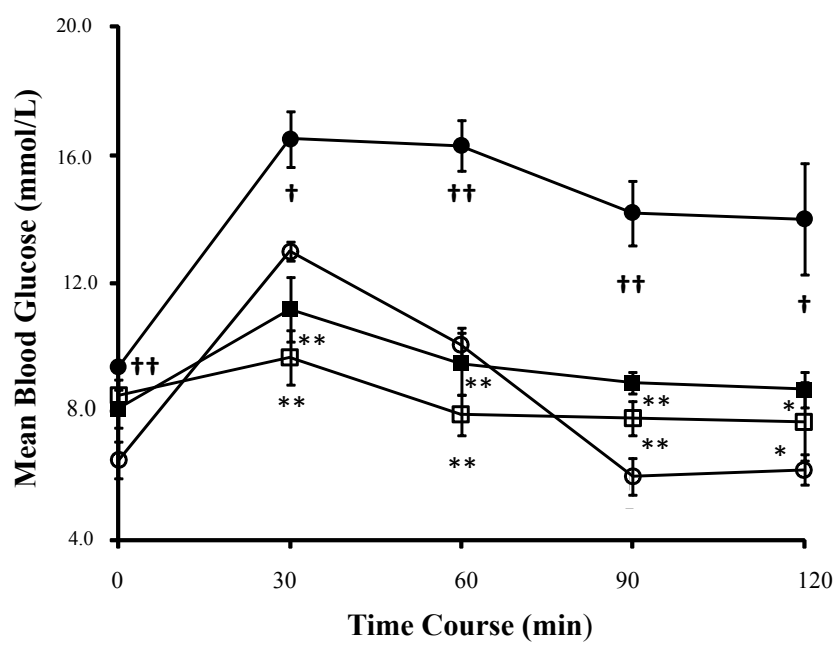

(b)

Figure 1. Effects of NI-SLN on OGTT in (a) normal rats and (b) diabetic rats. (NC,O); (DC, O); (NI, $\mathbf{\square})$; (NI-SLN, $\square$ ). The results are expressed as mean \pm SEM. $\dagger \mathrm{p}<0.05$ and $\dagger \dagger \mathrm{p}<0.01$ versus NC. ${ }^{\star} \mathrm{p}<0.05$ and ${ }^{\star *} \mathrm{p}<0.01$ versus DC.

Table 2. Time course of changes in blood glucose levels after 15 days of oral administration NI-SLN in fructose-induced diabetic rats.

\begin{tabular}{|c|c|c|c|c|}
\hline \multirow{2}{*}{ Group } & \multicolumn{4}{|c|}{ Mean Blood Glucose Level (mmol/L) } \\
\hline & 0 Day & 5 Day & 10 Day & 15 Day \\
\hline NC & $5.5 \pm 0.10$ & $6.0 \pm 0.24$ & $5.9 \pm 0.23$ & $5.4 \pm 0.64$ \\
\hline DC & $7.9 \pm 0.78 \dagger$ & $8.0 \pm 0.45 \dagger \dagger$ & $7.9 \pm 0.15+\dagger$ & $8.3 \pm 0.26+\dagger \dagger$ \\
\hline $\mathrm{NI}$ & $8.5 \pm 0.33+\dagger \dagger$ & $7.7 \pm 0.51$ & $7.1 \pm 0.42$ & $7.2 \pm 0.37^{*}$ \\
\hline NI-SLN 0.01 & $8.8 \pm 0.27 \dagger \dagger \dagger$ & $7.6 \pm 0.43$ & $7.3 \pm 0.25$ & $7.3 \pm 0.15^{\star}$ \\
\hline NI-SLN 0.1 & $8.7 \pm 1.00 \dagger$ & $7.0 \pm 0.78$ & $6.8 \pm 0.42^{\star}$ & $6.9 \pm 0.26^{* *}$ \\
\hline NI-SLN 1.0 & $8.7 \pm 0.32 \dagger \dagger \dagger$ & $6.7 \pm 0.35$ & $6.5 \pm 0.50^{*}$ & $6.2 \pm 0.35^{* *}$ \\
\hline
\end{tabular}

Data expressed as means \pm SEM. $\dagger \mathrm{p}<0.05, \dagger \dagger \mathrm{p}<0.01$ and $\dagger \dagger \dagger \mathrm{p}<0.001$ versus NC; ${ }^{*} \mathrm{p}<0.05,{ }^{* *} \mathrm{p}<0.01$ and ${ }^{* * *} \mathrm{p}<0.001$ versus DC. 
rats, no significant differences in blood glucose levels were observed among the treatment groups.

\subsubsection{Effects of NI-SLN on Body Weight and Organ Weight}

Body weights, organ weights and organ weight to body weight ratios are summarized in Table 3. The body weights and organ weights were higher in diabetic rats than that of NC rats. Although treatment with NI-SLN and NI lowered body weight, organ weight and organ weight to body weight ratio, the effects were not significant.

\subsubsection{Effects of NI-SLN on Lipid Profile}

The changes in lipid profile are shown in Table 4. The data revealed that the levels of TC, TG and LDL were significantly higher and HDL levels were significantly lower in diabetic rats as compared to NC rats. The increment of TC, TG, LDL and LDL/HDL ratio were attenuated and HDL level was increased after oral ingestion of NI-SLN. The Group NI-SLN 1.0 exhibited greater improvement in lipid profile among the treatment groups. Although NI decreased TC, TG, LDL and increased HDL, the effects were not significant.

\subsubsection{Effects of NI-SLN on SGOT and Total Bilirubin}

The liver enzyme SGOT and serum total bilirubin levels are shown in Table 4. The DC rats exhibited higher levels of SGOT and total bilirubin indicating inflammation and/or abnormal liver function. However, administration of both NI-SLN 1.0 and NI-SLN 0.1 significantly reduced SGOT levels and were comparable to that of NC rats. NI-SLN reduced total bilirubin levels but the effect was not significant.

\subsubsection{Effects of NI-SLN on Serum Uric Acid, Creatinine, Calcium and Electrolytes}

Table 4 represents the effects of NI-SLN on serum uric acid, creatinine, calcium and electrolytes. The data revealed that serum uric acid and creatinine levels were significantly elevated in DC rats. Administration of NI-SLN in diabetic rats reduced both uric acid and creatinine levels; and the effect of NI-SLN was dose-dependent. Furthermore, the serum levels of calcium and $\mathrm{K}^{+}$levels were significantly lower; and $\mathrm{Na}^{+}$and $\mathrm{Cl}^{-}$were significantly higher in DC rats. Administration of NI-SLN restored the levels of the serum calcium, $\mathrm{K}^{+}, \mathrm{Na}^{+}$and $\mathrm{Cl}^{-}$ and the effects were dose-dependent.

\subsubsection{Histopathology}

Representative photomicrographs of thin sections of liver stained with HE are presented in Figure 2. Liver from diabetic rats showed cytoplasmic inclusions with frosted appearance of hepatocytes due to intracellular accumulation of lipids and infiltrations indicating the presence of inflammations and damage. In group NC, hepatocytes were visualized with intact and normal cellular architecture. DC rats treated with NI and NI-SLN 0.01 showed partial clearance of lipids and attenuation of cellular swelling and inflammation. However, significant lipid clearance 
Table 3. Effects of NI-SLN on body weight, organ weight and organ weight to body weight ratios in fructose-induced diabetic rats.

\begin{tabular}{ccccccc}
\hline \multirow{2}{*}{ Parameters } & \multicolumn{7}{c}{ Groups } \\
\cline { 2 - 7 } & NC & DC & NI & NI-SLN 0.01 & NI-SLN 0.1 & NI-SLN 1.0 \\
\hline BW (g) & $235 \pm 5.69$ & $258 \pm 7.17$ & $241 \pm 6.73$ & $244 \pm 3.58$ & $236 \pm 9.87$ & $232 \pm 5.20$ \\
HW (g) & $0.73 \pm 0.02$ & $0.74 \pm 0.02$ & $0.73 \pm 0.03$ & $0.74 \pm 0.03$ & $0.73 \pm 0.03$ & $0.72 \pm 0.02$ \\
LW (g) & $7.6 \pm 0.10$ & $9.6 \pm 0.51$ & $8.2 \pm 0.54$ & $8.9 \pm 0.45$ & $8.3 \pm 0.54$ & $7.7 \pm 0.38$ \\
KW (g) & $1.6 \pm 0.04$ & $1.9 \pm 0.13$ & $1.7 \pm 0.09$ & $1.8 \pm 0.05$ & $1.7 \pm 0.04$ & $1.6 \pm 0.07$ \\
PW (g) & $0.50 \pm 0.04$ & $0.59 \pm 0.01$ & $0.54 \pm 0.05$ & $0.55 \pm 0.02$ & $0.52 \pm 0.02$ & $0.50 \pm 0.03$ \\
$\mathrm{HB}^{-1}(\mathrm{~g} / \mathrm{kg})$ & $3.1 \pm 0.04$ & $2.9 \pm 0.09$ & $3.0 \pm 0.08$ & $3.0 \pm 0.15$ & $3.1 \pm 0.21$ & $3.1 \pm 0.06$ \\
$\mathrm{LB}^{-1}(\mathrm{~g} / \mathrm{kg})$ & $32.4 \pm 0.44$ & $37.4 \pm 2.16$ & $34.1 \pm 1.31$ & $36.3 \pm 1.65$ & $34.9 \pm 1.45$ & $33.1 \pm 0.99$ \\
$\mathrm{~KB}^{-1}(\mathrm{~g} / \mathrm{kg})$ & $6.9 \pm 0.03$ & $7.4 \pm 0.38$ & $7.1 \pm 0.21$ & $7.2 \pm 0.26$ & $7.1 \pm 0.32$ & $7.0 \pm 0.16$ \\
$\mathrm{~PB}^{-1}(\mathrm{~g} / \mathrm{kg})$ & $2.1 \pm 0.13$ & $2.3 \pm 0.07$ & $2.3 \pm 0.15$ & $2.2 \pm 0.08$ & $2.2 \pm 0.11$ & $2.2 \pm 0.10$ \\
\hline
\end{tabular}

Data expressed as means \pm SEM. BW, body weight; HW, Heart weight; KW, kidney weight; PW, pancreas weight; $\mathrm{HB}^{-1}$, heart weight to body weight ratio; $\mathrm{LB}^{-1}$, liver weight to body weight ratio; $\mathrm{KB}^{-1}$, kidney weight to body weight ratio; $\mathrm{PB}^{-1}$, pancreas weight to body weight ratio. $\dagger \mathrm{p}<0.05 \mathrm{versus} \mathrm{NC}$; and ${ }^{*} \mathrm{p}<$ 0.05 versus DC.

Table 4. Effects of NI-SLN on serum lipid profile, uric acid, creatinine, calcium and electrolytes in fructose-induced diabetic rats.

\begin{tabular}{|c|c|c|c|c|c|c|}
\hline \multirow{2}{*}{ Parameters } & \multicolumn{6}{|c|}{ Groups } \\
\hline & $\mathrm{NC}$ & DC & NI & NI-SLN 0.01 & NI-SLN 0.1 & NI-SLN 1.0 \\
\hline $\mathrm{TC}(\mathrm{mg} / \mathrm{dL})$ & $87 \pm 3.11$ & $106 \pm 5.04 \dagger$ & $95 \pm 4.50$ & $90 \pm 4.96$ & $78 \pm 5.31^{* *}$ & $72 \pm 4.84^{* *}$ \\
\hline TG (mg/dL) & $88 \pm 4.25$ & $115 \pm 5.33+\dagger$ & $95 \pm 1.42^{*}$ & $99 \pm 4.68^{*}$ & $97 \pm 3.30^{*}$ & $84 \pm 4.03^{\star \star}$ \\
\hline $\mathrm{HDL}(\mathrm{mg} / \mathrm{dL})$ & $32.0 \pm 3.40$ & $25.1 \pm 2.20 \dagger$ & $29.5 \pm 1.62$ & $24.0 \pm 1.29$ & $31.6 \pm 1.28^{\star}$ & $38.1 \pm 1.02^{\star * *}$ \\
\hline LDL (mg/dL) & $37.0 \pm 3.40$ & $58.0 \pm 3.91 \dagger \dagger$ & $46.6 \pm 4.98$ & $46.5 \pm 5.47$ & $27.2 \pm 5.86^{\star *}$ & $17.1 \pm 4.75^{\star * *}$ \\
\hline LDL/HDL & $1.16 \pm 0.11$ & $2.41 \pm 0.31 \dagger \dagger$ & $1.63 \pm 0.24$ & $1.97 \pm 0.26$ & $0.89 \pm 0.22^{* *}$ & $0.45 \pm 0.12^{* *}$ \\
\hline SGOT (mg/dL) & $20.5 \pm 2.17$ & $40.0 \pm 3.22 \dagger \dagger$ & $33.2 \pm 3.31$ & $36.9 \pm 2.95$ & $24.1 \pm 2.38^{\star *}$ & $21.6 \pm 2.65^{* *}$ \\
\hline Bilirubin (mg/dL) & $0.56 \pm 0.04$ & $0.85 \pm 0.07 \dagger+\dagger$ & $0.79 \pm 0.03$ & $0.81 \pm 0.04$ & $0.76 \pm 0.04$ & $0.67 \pm 0.04$ \\
\hline Uric Acid (mg/dL) & $3.5 \pm 0.47$ & $5.2 \pm 0.49 \dagger$ & $4.8 \pm 0.47$ & $4.7 \pm 0.62$ & $4.5 \pm 0.51$ & $3.9 \pm 0.23^{\star}$ \\
\hline Creatinine (mg/dL) & $0.56 \pm 0.09$ & $1.12 \pm 0.17 \dagger$ & $0.9 \pm 0.13$ & $0.86 \pm 0.12$ & $0.78 \pm 0.12$ & $0.64 \pm 0.10^{*}$ \\
\hline Calcium (mg/dL) & $8.6 \pm 0.24$ & $6.8 \pm 0.29 \dagger \dagger$ & $7.3 \pm 0.21$ & $7.2 \pm 0.27$ & $7.5 \pm 0.22$ & $7.7 \pm 0.29$ \\
\hline $\mathrm{Na}^{+}(\mathrm{mmol} / \mathrm{L})$ & $133 \pm 3.41$ & $151 \pm 6.40 \dagger$ & $135 \pm 2.16$ & $137 \pm 4.81$ & $133 \pm 3.71^{*}$ & $129 \pm 4.50^{*}$ \\
\hline $\mathrm{K}^{+}(\mathrm{mmol} / \mathrm{L})$ & $5.2 \pm 0.19$ & $4.6 \pm 0.02 \dagger$ & $4.5 \pm 0.21$ & $4.1 \pm 0.20$ & $4.7 \pm 0.29$ & $5.1 \pm 0.25$ \\
\hline $\mathrm{Cl}^{-}(\mathrm{mmol} / \mathrm{L})$ & $94 \pm 1.02$ & $104 \pm 0.82 \dagger \dagger \dagger$ & $102 \pm 2.70$ & $95 \pm 1.14^{* *}$ & $94 \pm 1.80^{* *}$ & $92 \pm 2.09^{\star * *}$ \\
\hline
\end{tabular}

Data expressed as means \pm SEM. $\dagger \mathrm{p}<0.05, \dagger \dagger \mathrm{p}<0.01$ and $\dagger \dagger \dagger \mathrm{p}<0.001$ versus NC; ${ }^{*} \mathrm{p}<0.05,{ }^{* *} \mathrm{p}<0.01$ and ${ }^{* * *} \mathrm{p}<0.001$ versus DC.

and reduction of inflammations were observed in Groups NI-SLN 0.1 and NI-SLN 1.0 which can be correlated with the significant attenuation of SGOT levels by the treatment suggestive of improvement in liver function and morphology.

\section{Discussion}

Insulin resistance and hyperinsulinemia are frequently associated with both 

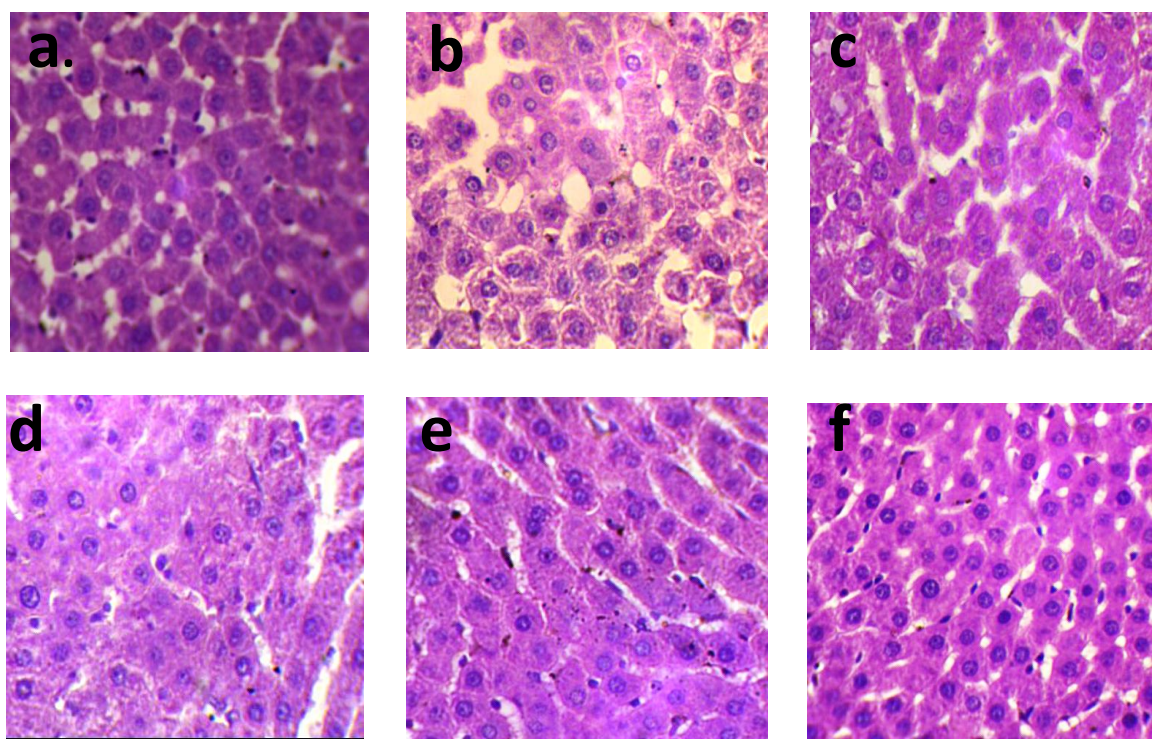

Figure 2. Effects of NI and NI-SLN on liver histopathology. HE staining of the cross sectional tissues of liver (original magnifications 400X). (a) Group NC, age-matched normal rats; showing intact hepatocytes with normal cellular architecture. (b) Group DC, diabetic control rats treated with vehicle; depicting lipid deposition, inflammations and damage in liver. (c) Group NI, diabetic rats treated with NI $(0.1 \mathrm{mg} / \mathrm{kg})$; showing presence of intracellular accumulation of lipids and partial attenuation of infiltrations and damage. (d) Group NI-SLN 0.01, diabetic rats treated with low dose of NI-SLN $(0.01 \mathrm{mg} / \mathrm{kg})$; showing partial clearance of lipids, attenuation of cellular swelling and inflammations (e) Group NI-SLN 0.1, diabetic rats treated with medium dose of NI-SLN $(0.1 \mathrm{mg} / \mathrm{kg})$; showing mild to moderate clearance of lipids and reduction of inflammations. (f) Group NI-SLN 1.0, diabetic rats treated with high dose of NI-SLN $(1.0 \mathrm{mg} / \mathrm{kg})$, showing normal liver architecture with reduction of inflammation and damage.

clinical and experimental hypertension [18]. In dietary rodent models, CCBs can attenuate many of the symptoms of metabolic syndrome [19] [20]. Both dihydropyridine and long-acting nondihydropyridine CCBs have shown metabolic benefits, with effects on insulin sensitivity and insulin secretion. Also, ACE-Is and ARBs were shown to reduce insulin resistance while thiazide diuretics and $\beta$-blockers may complicate the conditions [21]. NI is a CCB known to be metabolically neutral which implies it has no effect on glucose homeostasis or lipid metabolism [22]. However, recent studies indicated that NI is effective in reducing plasma glucose and serum TG levels in hypertensive patients along with the capacity of increasing the plasma HDL level [12]. NI is widely used as an anti-hypertensive. However, the use of this drug is limited due to its poor aqueous solubility despite its high membrane permeability. Recently, our co-workers reported that the aqueous solubility of NI increased by about 4 -fold when loaded in SLN [5]. In addition, NI is known to possess antioxidant properties and may improve insulin sensitivity by increasing insulin uptake by tissue for glucose metabolism [23]. Therefore, the present study was designed to evaluate the effects of a newly developed NI-SLN on glucose homeostasis, lipid metabolism in states of metabolic syndrome in fructose-induced diabetic rats. 
We have developed NI-SLN lyophilized with trehalose (NI-SLN-Tre) that showed a good pharmacokinetic profile, as well as the NI-SLN suspension. The $C_{\max }, A U C_{0 \rightarrow \infty}$ and $t_{1 / 2}$ values of the NI-SLN-Tre were found to be same to those of the NI-SLN, and NI tended to be absorbed to a much greater extent from the lyophilized NI-SLN-Tre suspension because of the enhanced solvation of NI-SLN in gastrointestinal fluid derived from formation of hydrogen bonds between the polar head groups of the lipids and the $\mathrm{O}-\mathrm{H}$ groups of trehalose. Moreover, the hemolysis assay revealed that the NI-SLN and NI-SLN-Tre suspensions showed good hemocompatibility properties [5] [6]. Our results demonstrated that NI and NI-SLN treatment did not alter blood glucose level in normal rats. Blood glucose levels were significantly reduced in fructose-induced diabetic rats over $24 \mathrm{hr}$ after treatment with NI-SLN. In contrast, NI treated rats showed decrease in blood glucose levels, however, the effect was not significant. The effect of NI-SLN and NI on changes in $24 \mathrm{hr}$ plasma glucose levels was in accordance with the results of Sheu et al., who showed that the day-long glycemic response was modestly lower after NI therapy in patients with hypertension [12]. In OGTT, NI could not inhibit hyperglycemia whereas NI-SLN significantly exhibited anti-hyperglycemic activity in NC rats. In contrast, both NI and NI-SLN showed improvement in glucose tolerance in diabetic rats. These findings thus, imply that NI-SLN appears to counteract this effect of hyperglycemia in both normal and diabetic rats, whereas NI found only to show some effectiveness in diabetic rats. Similar observations were obtained by Kito et al. indicated that CCBs did not significantly affect plasma glucose levels in normal rats; however, they were effective in attenuation of high glucose levels in diabetic rats [20].

After 15 days, the survival rate in rats among the treatment group was 100\%. None of the rats died in NI, NI-SLN and vehicle-treated groups. Normal rats treated with NI-SLN 1.0 showed significant reduction $(\mathrm{p}<0.05)$ in blood glucose levels, however, NI did not exhibit any significant alterations in glucose levels. A peripheral vasoconstriction could account for both high glucose level and impaired insulin sensitivity in fructose-fed rats. However, NI-SLN was able to prevent fructose induced hyperglycemia in rats may be due to vasodilatory action of NI. Further, fructose feeding causes characteristics of obesity in rats that is increased body weight, hyperlipidemia and hypertriglyceridemia [24] [25]. After 15 days of oral administration, NI-SLN lowered body weights, organ weights and organ weight to body weight ratios. Treatment with NI-SLN showed a significant decrease in TC, TG, LDL and LDL/HDL ratio with increased HDL than that of NI. Recent, studies indicated that felodipine, a dihydropyridine CCB, which showed a significant reduction in the levels of serum TC, TG and LDL-cholesterol in patients with hypertension [26]. The mechanism by which NI lowered lipid levels in diabetic rats might be resulted from the activation of cellular cholesterol ester hydrolase [27]. Moreover, hormone-sensitive triglyceride lipase and LDL-cholesterol receptors played a significant role in lowering TG and LDL-cholesterol [28] [29]. 
In the current study, treatment with NI-SLN showed significant reduction in SGOT, uric acid and creatinine levels whereas NI did not. Elevated serum uric acid predicts the development of insulin resistance, obesity and hypertension [22]. NI-SLN treatment reduced uric acid levels which might be partly responsible for improving insulin sensitivity and subsequent hypertriglyceridemia in fructose-induced diabetic rats [30] [31]. Diabetic rats exhibited cationic imbalances and our observations were in accordance with study reported earlier [32]. Treatment with NI-SLN increased the concentration of serum calcium and $\mathrm{K}^{+}$ with a decreased the concentration of serum $\mathrm{Na}^{+}$and $\mathrm{Cl}^{-}$. Acute role of systemic calcium has invoked its function in insulin secretion, appropriate response to glucose and hypertension [33]. Administration of NI-SLN has resulted in significant increase in serum calcium which may be responsible for insulin release by exocytosis which further contributes to the improvement in glucose metabolism [34]. Liver from diabetic rats showed cellular accumulation of lipids and inflammations as evidenced by increased in liver weight and SGOT levels. Hepatotoxicity is associated with abnormal calcium homeostasis, which may result in the activation of many membrane damaging enzymes like ATPases, phospholipases, proteases and endonucleases; and thus causes disruption of mitochondrial energy metabolism and ultimately damage of cellular architecture [35] [36]. Although, NI alone could not preserve liver structure in fructose-induced diabetic rats; administration of NI-SLN reduced SGOT levels and hepatic damage. The results obtained in this study suggested that NI-SLN might have hepatoprotective activity owing to the improvement of diabetic conditions.

\section{Conclusion}

NI-SLN was prepared by high-pressure homogenization technique followed by lyophilization with trehalose; and it was converted into suitable oral suspension useful for enhancement of bioavailability and in vivo evaluation. Administration of NI-SLN improved glucose tolerance, lipid profiles and liver function better than the conventional NI in fructose-fed rats. So, NI-SLN played an important role in the treatment of impaired glucose tolerance, insulin resistance and hypertriglyceridemia in fructose-induced diabetic rats. A peripheral vasoconstriction and elevation in serum uric acid level could be responsible for diminished insulin sensitivity and hypertriglyceridemia in fructose-induced diabetic rats. The mechanism by which NI-SLN improved insulin sensitivity and dyslipidemia could be partly explained by the reduction of uric acid level and enhanced vasodilatory actions of it. So, this newer formulation NI-SLN might be safe and effective alternative to conventional dosage forms of NI in diabetic patients with poor lipid control. However, further pharmacological and biochemical studies are warranted to elucidate the exact mechanism of actions of NI-SLN in experimental animal model. In addition, the delivery system might be safe and an effective alternative to the commercially available dosage forms 
in near future.

\section{Acknowledgements}

This research was supported by National Science and Technology (NST) fellowships offered by the Ministry of Science and Technology (MOST), Bangladesh.

\section{Conflicts of Interest}

The authors declare that they have no competing interests.

\section{References}

[1] Kohli, K., Sunny, C., Deepika, D., Saurabh, A. and Roop, K.K. (2010) Self-Emulsifying Drug Delivery Systems: An Approach to Enhance Oral Bioavailability. Drug Discovery Today, 15, 958-965. https://doi.org/10.1016/j.drudis.2010.08.007

[2] Kim, Y.I., Fluckiger, L., Hoffman, M., Lartaud-Idjouadiene, I., Atkinson, J. and Maincent, T. (1997) The Antihypertensive Effect of Orally Administered Nifedipine-Loaded Nanoparticles in Spontaneously Hypertensive Rats. British Journal of Pharmacology, 120, 399-404. https://doi.org/10.1038/sj.bjp.0700910

[3] Raemsch, K.D. and Sommer, J. (1983) Pharmacokinetics and Metabolism of Nifedipine. Hypertension, 5, 1118-1124. https://doi.org/10.1161/01.HYP.5.4_Pt_2.II18

[4] Pabst, G., Lutz, D., Moltz, K.H., Dahmen, W. and Jaeger, H. (1986) Pharmakinetics and Bioavailability of Three Different Galenic Nifedipine Preparations. Arzneimittel-Forschung/Drug Research, 36, 256-260.

[5] Barman, R.K., Iwao, Y., Funakoshi, Y., Ranneh, A.H., Noguchi, S., Wahed, M.I. and Itai, S. (2014) Development of Highly Stable Nifedipine Solid-Lipid Nanoparticles. Chemical and Pharmaceutical Bulletin, 62, 399-406. https://doi.org/10.1248/cpb.c13-00684

[6] Barman, R.K., Iwao, Y., Islam, M.R., Funakoshi, Y., Noguchi, S., Wahed, M.I. and Itai, S. (2014) In Vivo Pharmacokinetic and Hemocompatible Evaluation of Lyophilization Induced Nifedipine Solid-Lipid Nanoparticle. Pharmacology \& Pharmacy, 5, 455-461. https://doi.org/10.4236/pp.2014.55055

[7] Funakoshi, Y., Iwao, Y., Noguchi, S. and Itai, S. (2013) Lipid Nanoparticles with No Surfactant Improve Oral Absorption Rate of Poorly Water-Soluble Drug. International Journal of Pharmaceutics, 451, 92-94. https://doi.org/10.1016/j.ijpharm.2013.04.050

[8] Lithell, H.O. (1991) Effects of Antihypertensive Drugs on Insulin, Glucose and Lipid Metabolism. Diabetes Care, 14, 203-209. https://doi.org/10.2337/diacare.14.3.203

[9] Navarro-Cid, J., Maeso, R., Perez-Vizcaino, F., Cachofeiro, V., Ruilope, L.M., Tamargo, J. and Lahera, V. (1995) Effects of Losartan on Blood Pressure, Metabolic Alterations, and Vascular Reactivity in the Fructose-Induced Hypertensive Rat. Hypertension, 26, 1074-1078. https://doi.org/10.1161/01.HYP.26.6.1074

[10] Kaplan, N.M. (1992) Effects of Antihypertensive Therapy on Insulin Resistance. Hypertension, 19, I-116-I-118. https://doi.org/10.1161/01.HYP.19.1_Suppl.I116

[11] Klauser, R., Prager, R., Gaube, S., Gisinger, C., Schnack, C., Küenburg, E. and Schernthaner, G. (1991) Metabolic Effects of Isradipine versus Hydrochlorothiazide in Diabetes Mellitus. Hypertension, 17, 15-21. https://doi.org/10.1161/01.HYP.17.1.15 
[12] Sheu, W.H., Swislocki, A.M., Hoffman, B., Chen, Y.D. and Reaven, G.M. (1991) Comparison of the Effects of Atenolol and Nifedipine on Glucose, Insulin, and Lipid Metabolism in Patients with Hypertension. American Journal of Hypertension, 4, 199-205. https://doi.org/10.1093/ajh/4.3.199

[13] Hwang, I.S., Ho, H.E., Hoffman, B.B. and Reaven, G.M. (1987) Fructose-Induced Insulin Resistance and Hypertension in Rats. Hypertension, 10, 512-516. https://doi.org/10.1161/01.HYP.10.5.512

[14] Dai, S. and McNeill, J.H. (1995) Fructose-Induced Hypertension in Rats Is Concentration and Duration-Dependent. Journal of Pharmacological and Toxicological Methods, 33, 101-107. https://doi.org/10.1016/1056-8719(94)00063-A

[15] Bergmeyer, H.U. and Bernt, E. (1974) Determination of Glucose with Glucose Oxidase and Peroxidase. Methods of Enzymatic Analysis, 2, 1205-1215.

[16] Morton, D.B., Abbot, D., Barclay, R., Close, B.S., Ewbank, R., Gask, D., Heath, M., Mattic, S., Poole, T., Seamer, J. and Southee, J. (1993) Removal of Blood from Laboratory Mammals and Birds. Laboratory Animals, 27, 1-22. https://doi.org/10.1258/002367793781082412

[17] Fukuyama, N., Homma, K., Wakana, N., Kudo, K., Suyama, A., Ohazama, H., Tsuji, C., Ishiwata, K., Eguchi, Y., Nakazawa, H. and Tanaka, E. (2008) Validation of the Friedewald Equation for Evaluation of Plasma LDL-Cholesterol. Journal of Clinical Biochemistry and Nutrition, 43, 1-5. https://doi.org/10.3164/jcbn.2008036

[18] Verma, S., Bhanot, S. and McNeill, J. (1994) Antihypertensive Effects of Metformin in Fructose-Fed Hyperinsulinemic, Hypertensive Rats. Journal of Pharmacology and Experimental Therapeutics, 271, 1334-1337.

[19] Kito, T., Shibata, R., Kondo, M., Yamamoto, T., Suzuki, H., Ishii, M. and Murohara, T. (2012) Nifedipine Ameliorates Ischemia-Induced Revascularization in Diet-Induced Obese Mice. American Journal of Hypertension, 25, 401-406. https://doi.org/10.1038/ajh.2011.239

[20] Navarro-Cid, J., Maeso, R., Perez-Vizcaino, F., Casal, M.C., Cachofeiro, V., Ruilope, L.M. and Lahera, V. (1996) Effects of Antihypertensive Drugs on Blood Pressure and Metabolic Alterations in the Fructose-Induced Hypertensive Rat. American Journal of hypertension, 9, 669-674. https://doi.org/10.1016/0895-7061(96)00024-6

[21] Pepine, C.J., Handberg, E.M., Cooper-DeHoff, R.M., Marks, R.G., Kowey, P., Messerli, F.H., Mancia, G., Cangiano, J.L., Garcia-Barreto, D., Keltai, M. and Erdine, S. (2003) A Calcium Antagonist vs. a Non-Calcium Antagonist Hypertension Treatment Strategy for Patients with Coronary Artery Disease: The International Verapamil-Trandolapril Study (INVEST): A Randomized Controlled Trial. JAMA, 290, 2805-1286. https://doi.org/10.1001/jama.290.21.2805

[22] Masuo, K., Kawaguchi, H., Mikami, H., Ogihara, T. and Tuck, M.L. (2003) Serum Uric Acid and Plasma Norepinephrine Concentrations Predict Subsequent Weight Gain and Blood Pressure Elevation. Hypertension, 42, 474-480. https://doi.org/10.1161/01.HYP.0000091371.53502.D3

[23] Sobal, G., Menzel, E.J. and Sinzinger, H. (2001) Calcium Antagonists as Inhibitors of in Vitro Low Density Lipoprotein Oxidation and Glycation. Biochemical Pharmacology, 61, 373-379. https://doi.org/10.1016/S0006-2952(00)00548-7

[24] Bocarsly, M.E., Powell, E.S., Avena, N.M. and Hoebel, B.G. (2010) High-Fructose Corn Syrup Causes Characteristics of Obesity in Rats: Increased Body Weight, Body Fat and Triglyceride Levels. Pharmacology Biochemistry and Behavior, 97, 101-106. https://doi.org/10.1016/j.pbb.2010.02.012

[25] Girard, A., Sihem, M., Boukortt, F., Cherkaoui-Malki, M., Belleville, J. and Prost, J. 
(2006) Fructose-Enriched Diet Modifies Antioxidant Status and Lipid Metabolism in Spontaneous Hypertensive Rats. Nutrition, 22, 758-766. https://doi.org/10.1016/j.nut.2006.05.006

[26] Kaur, M., Kaur, K., Bedi, G.K., Sidhu, G.S. and Sikand, R. (2000) Effect of Felodipine on the Serum Lipid Profile of Patients with Hypertension. Indian Journal of Clinical Biochemistry, 15, 63-67. https://doi.org/10.1007/BF02883730

[27] Eltingh, O.P. and Hajjar, D.P. (1985) Nifedipine Increases Cholesteryl Ester Hydrolytic Activity in Lipid Laden Arterial Smooth Muscle Cells. Journal of Clinical Investigation, 15, 1554-1558.

[28] Klein, W.W. (1984) Treatment of Hypertension with Calcium Channel Blockers-European Data. American Journal of Medicine, 76, 143-146. https://doi.org/10.1016/S0002-9343(84)80050-9

[29] Fillipovic, I. and Buddecke, E. (1986) Calcium Channel Blockers Stimulate LDL Receptor Synthesis in Human Skin Fibroblasts. Biochemical and Biophysical Research Communications, 136, 845-850. https://doi.org/10.1016/0006-291X(86)90409-2

[30] Nakagawa, T., Tuttle, K.R., Short, R.A. and Johnson, R.J. (2005) Hypothesis: Fructose-Induced Hyperuricemia as a Causal Mechanism for the Epidemic of the Metabolic Syndrome. Nature Clinical Practice Nephrology, 1, 80-86. https://doi.org/10.1038/ncpneph0019

[31] Nakagawa, T., Hu, H., Zharikov, S., Tuttle, K.R., Short, R.A., Glushakova, O., Ouyang, X., Feig, D.I., Block, E.R., Herrera-Acosta, J., Patel, J.M. and Johnson, R.J. (2006) A Casual for Uric Acid in Fructose Induced Metabolic Syndrome. American Journal of Physiology-Renal Physiology, 290, F625-F631. https://doi.org/10.1152/ajprenal.00140.2005

[32] Haebisch, E.B. (1988) Nifedipine, Its Action on the Cationic Concentrations in Heart, Vessels, Skeletal Muscle and Blood in Tissues of Normotensive and Spontaneously Hypertensive Rats (SHR). General Pharmacology. The Vascular System, 19, 407-416. https://doi.org/10.1016/0306-3623(88)90038-9

[33] Friel, K. (2004) The Effects and Interactions of Vitamin D Deficiency, Calcium and Parathyroid Hormone on Physical Concerns in the Elderly: A Review. Journal of Geriatric Physical Therapy, 27, 30-34. https://doi.org/10.1519/00139143-200404000-00005

[34] Wollheim, C.B. and Sharp, G.W. (1981) Regulation of Insulin Release by Calcium. Physiological Reviews, 61, 914-973. https://doi.org/10.1152/physrev.1981.61.4.914

[35] Singh, A., Bhat, T.K. and Sharma, O.P. (2011) Clinical Biochemistry of Hepatotoxicity. Journal of Clinical Toxicology, S4, 001.

[36] Okwa, I.B., Akindele, A.J., Agbaje, E.O., Oshinuga, O.T., Anunobi, C.C. and Adeyemi, O.O. (2013) Effect of Subclinical, Clinical and Supraclinical Doses of Calcium Channel Blockers on Models of Drug-Induced Hepatotoxicity in Rats. EXCLI Journal, 12, 231-250. 